\title{
GLAD!
}

Revue sur le langage, le genre, les sexualités

03 | 2017

Hétérosexualités

\section{Les Quotas}

Quotas

\section{Marie-Laure Schultze et Sujarei Tali}

\section{(2) OpenEdition}

Journals

Édition électronique

URL : http://journals.openedition.org/glad/909

DOI : $10.4000 /$ glad. 909

ISSN : 2551-0819

\section{Éditeur}

Association GSL

\section{Référence électronique}

Marie-Laure Schultze et Sujarei Tali, «Les Quotas », GLAD! [En ligne], 03 | 2017, mis en ligne le 10 décembre 2017, consulté le 20 janvier 2021. URL : http://journals.openedition.org/glad/909 ; DOI : https://doi.org/10.4000/glad.909

Ce document a été généré automatiquement le 20 janvier 2021.

\section{(c) (i) (9)}

La revue GLAD! est mise à disposition selon les termes de la Licence Creative Commons Attribution Pas d'Utilisation Commerciale - Pas de Modification 4.0 International. 


\title{
Les Quotas
}

\author{
Quotas
}

\section{Marie-Laure Schultze et Sujarei Tali}

1 Le texte Les Quotas ${ }^{1}$ est le premier d'une série dont le deuxième a été publié chez un petit éditeur, Griffe d'Encre (ce texte, L'Anémone, est désormais accessible sur mon Carnet de Recherches ${ }^{2}$ ). J'avais été mise en contact avec une des éditrices, elle cherchait des textes de SFFF (science fiction, fantasy \& fantastique) érotique. C'était tentant : ce que j'avais pu lire en termes d'“érotisme" alors que je travaillais à ma thèse de doctorat sur l'heroic fantasy anglophone dans les années 1990 dépassait rarement le stade du «toi, femme, moi le guerrier je te veux, couche-toi et écarte les cuisses et ne dis pas non ; quand une femme dit non, elle dit oui », accompagné de plus ou moins de violence à l'égard de la femme. Je n'avais peut-être pas lu les bons textes : en gros, dans ceux que j'avais lus, les lecteurs n'échappaient pas aux variations sur Mère ou Putain.

2 À l'occasion de cette proposition de textes de SFFF érotique, j'ai ouvert un univers dystopique qui continue à se développer ${ }^{3}$. Ce qu'on en voit se passe sur un astronef miteux de fond de galaxie où l'Intergalactique, le pouvoir totalitaire en place, traque ceux qui ne se soumettent pas à ses lois. Parmi celles-ci, l'obligation de se livrer à des coïts dont le nombre par période de temps est fixé par l'Inter. Le choix de avec qui, comment et surtout à quel rythme vous avez des relations sexuelles, dans cet univers, ne vous appartient pas.

Dans Les Quotas, Kimée est sur l'astronef pourri dans l'espoir de rencontrer quelqu'un avec qui avoir un coït car coïts insuffisants = sanctions variées. Kimée a ainsi déjà eu ses rations d'énergie supprimées ; l'autre personnage, Djoa, a subi des mutilations.

4 Tous les textes de la série sont des témoignages de contrevenants aux divers règlements imposés par l'Inter. Ces contrevenants témoignent devant des " Résistants » qui les enlèvent pour recueillir un maximum d'informations sur les agissements de l'Inter. Les contrevenants peuvent avoir également trouvé refuge chez ces Résistants et échanger leur témoignage contre des services (dans L'Anémone, il s'agit pour le personnage de se faire ôter l'anémone très sensible qui lui est sortie au niveau de 
l'anus). Les lecteurs n'apprennent pas grand chose des Résistants. En réalité, ils ont l'impression que c'est à eux que les témoins s'adressent.

5 L'enjeu de cet univers est évidemment la connaissance : qui peut apprendre et savoir quoi. C'est un univers banalement post-apocalyptique dont la civilisation des "Anciens» a été pratiquement anéantie par un cataclysme. Il ne reste d'eux que des bribes de langage - dans Les Quotas, des expressions comme " aller à confesse ", " avoir le diable au corps » - et, peut-être, des informations sur les Plaquettes d'Instruction, équivalents de manuels scolaires et universitaires pour les plus rudimentaires. Mais l'Inter contrôle une grande partie de ces plaquettes et il est très difficile de savoir si elles contiennent des informations justes, ou bien la propagande de l'Inter.

Un des enjeux du texte était pour moi d'écrire sans marques grammaticales de genre, ce qui en français est difficile, afin que les personnages puissent être hermaphrodites. La question est vieille comme le monde : qu'en serait-il de la " guerre des sexes ", et des relations de pouvoir, si nous étions tous hermaphrodites?

7 Plus tard, j'ai su que Julia, que j'avais croisée alors que j'étais enseignante et elle étudiante, dessinait. Je lui ai demandé si elle aimerait illustrer un de mes textes. J'avais plutôt mes textes pour enfants à l'esprit, mais elle a choisi Les Quotas. La suite est son histoire.

\section{Les Quotas, une lecture sonore}

Texte: Marie-Laure Schultze ; Voix: Épicène et Vlan
Ce média ne peut être affiché ici. Veuillez vous reporter à l'édition en ligne http://
journals.openedition.org/glad/909

\section{Les Quotas, une bande dessinée}


Les Quotas

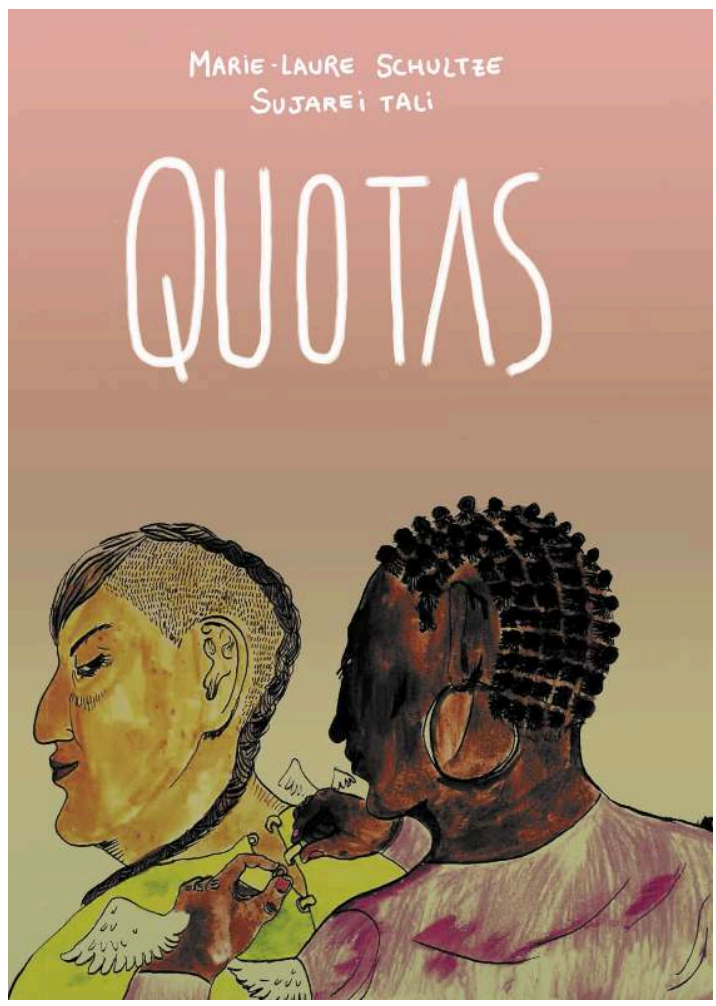

Ça s'annonçait mal

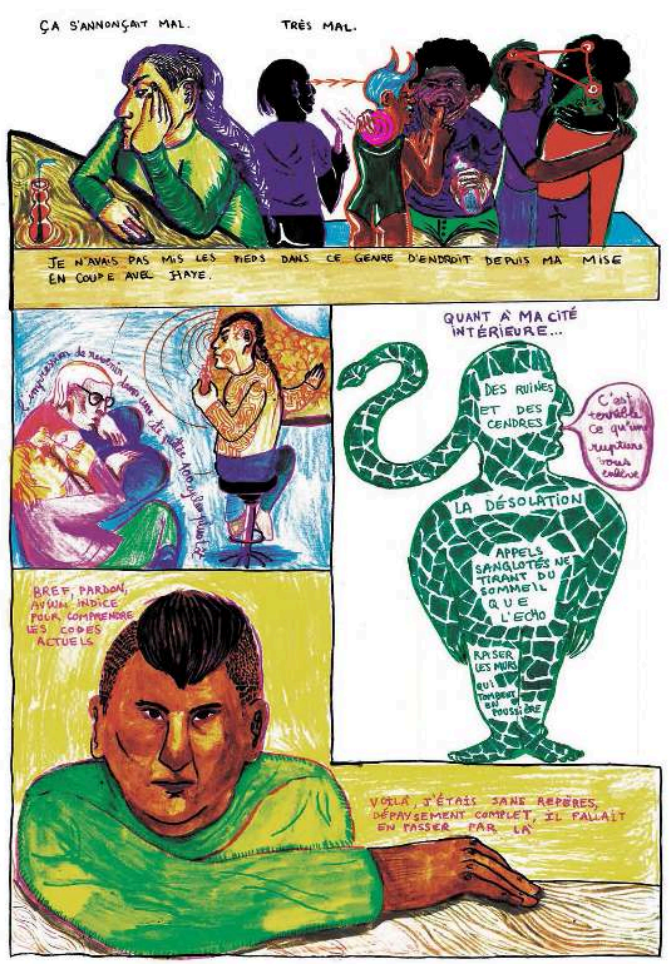


Si au moins Haye avait accepté qu'on fasse semblant encore quelques cycles

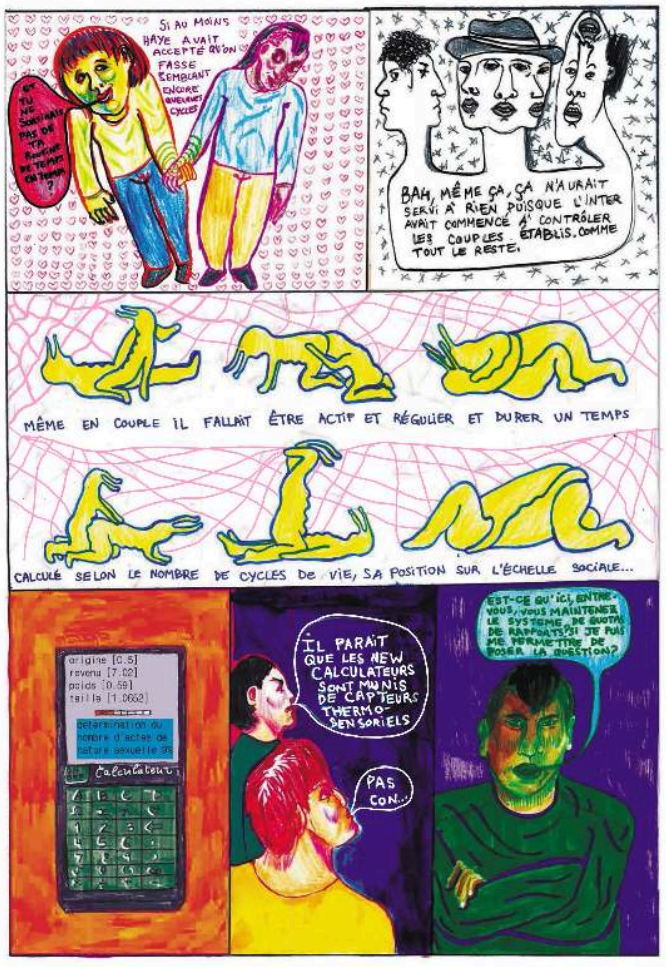

Un dernier nanoblast

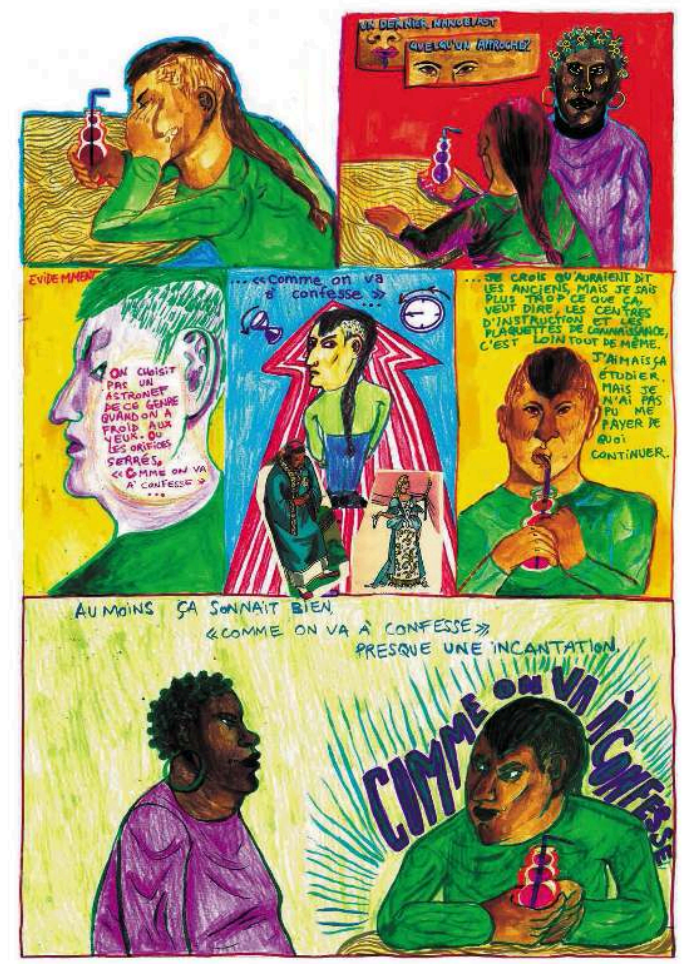


Bonsoir, je fonctionne sous le nom de Djoa

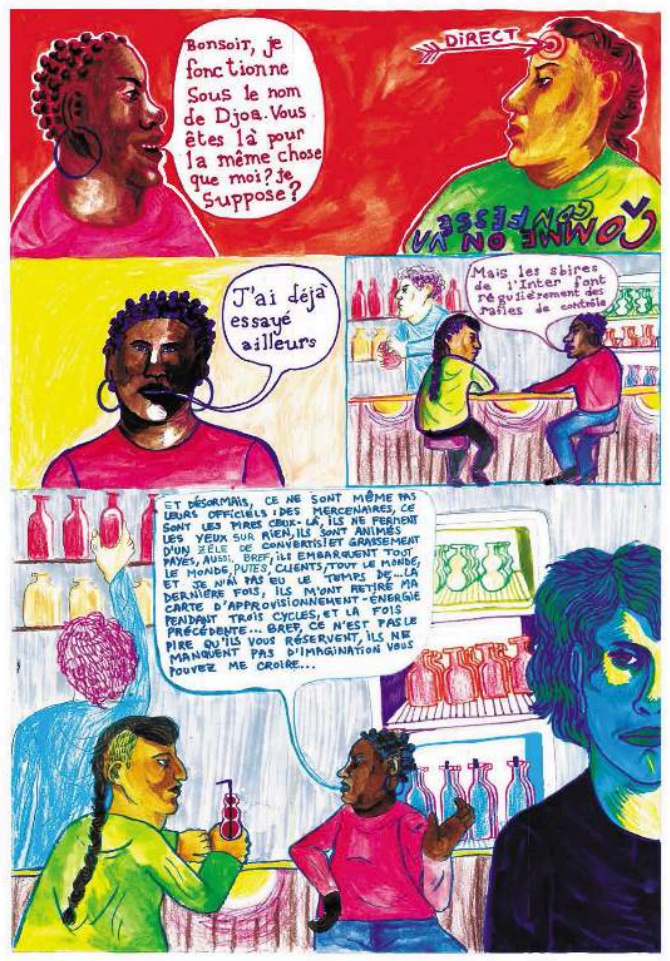

Sur le comptoir...

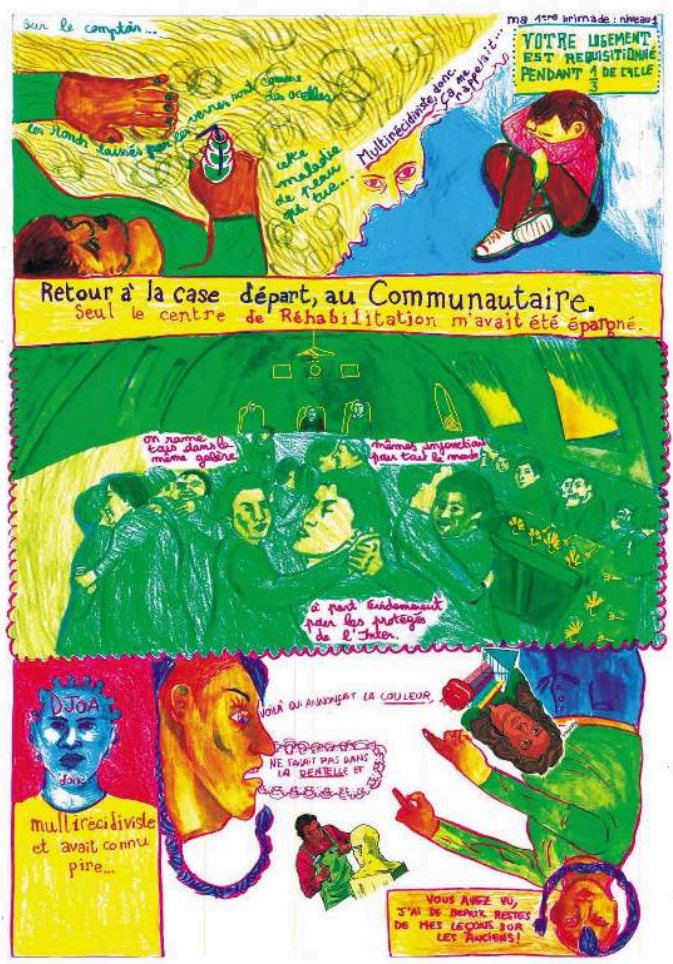


Bref, c'était à peine croyable mais j'avais le désir qui s'éveillait

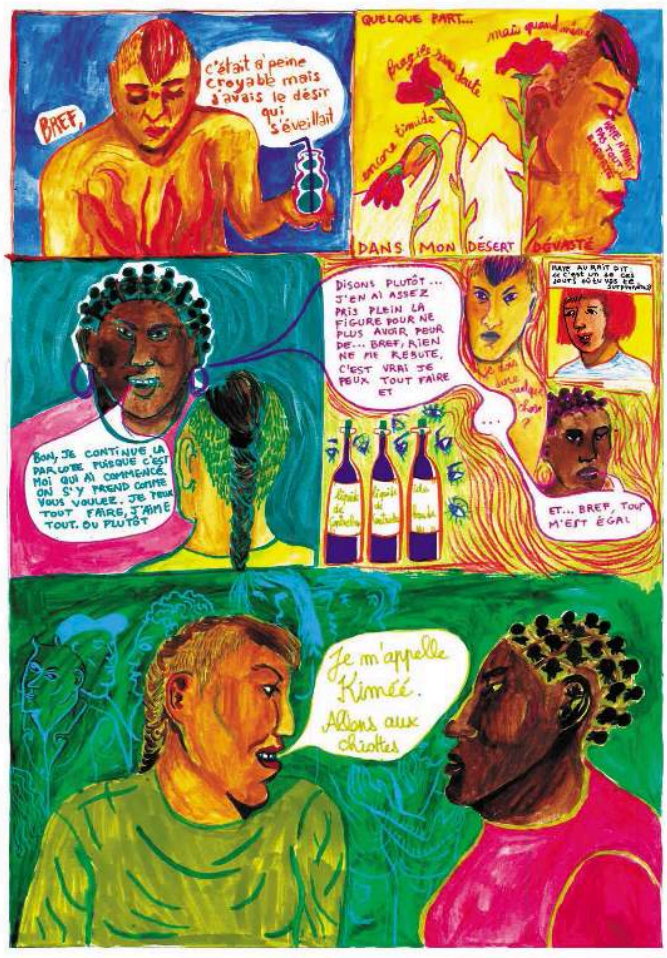

Déjà ? Vous ne voulez pas boire un verre?

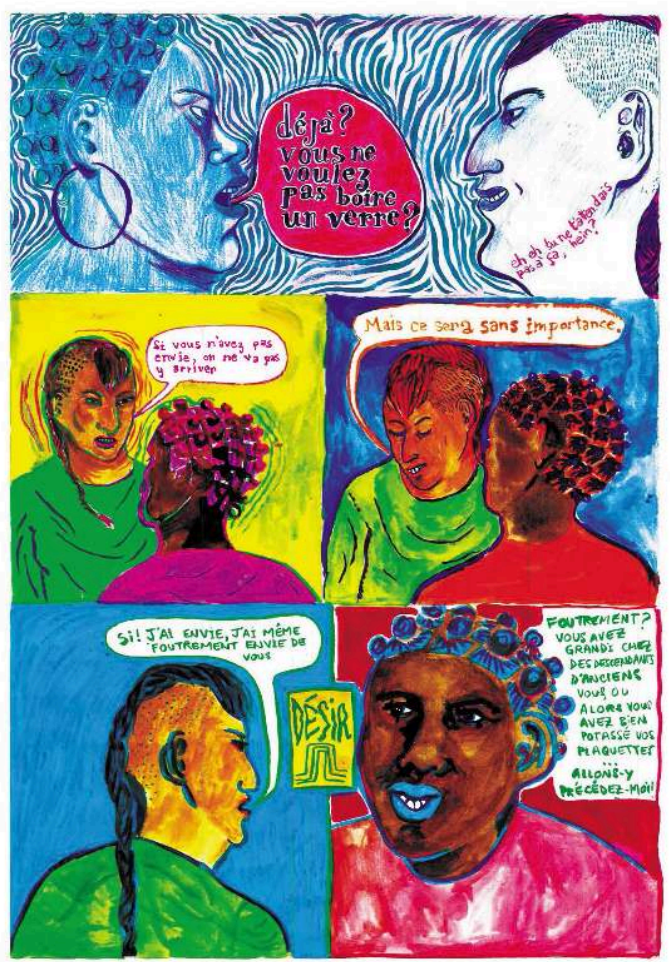


J'ai choisi les toilettes handicapés

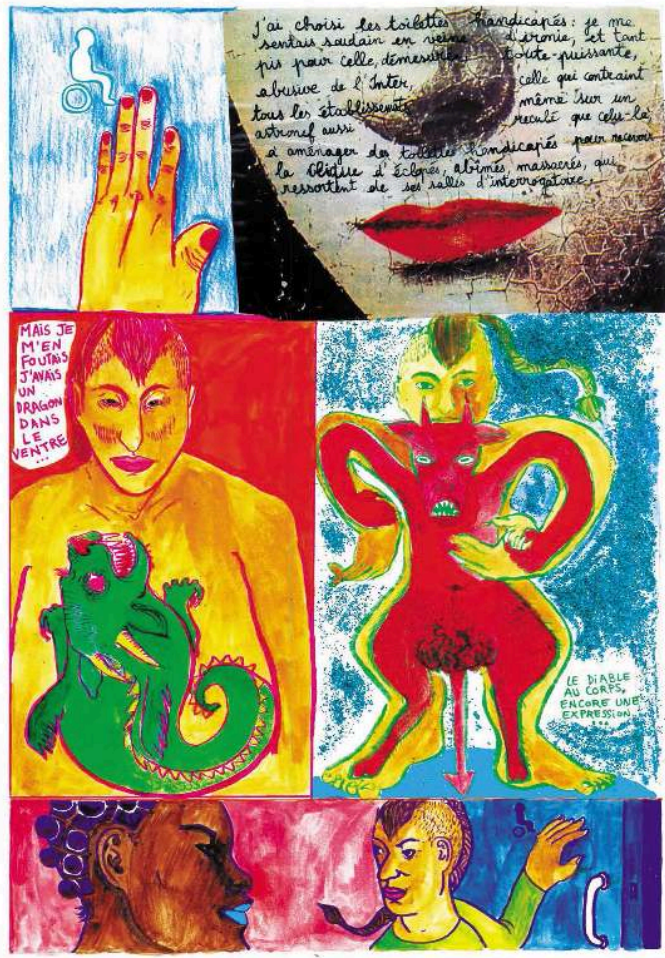

C'est ça ou pas d'énergie pendant trois cycles.

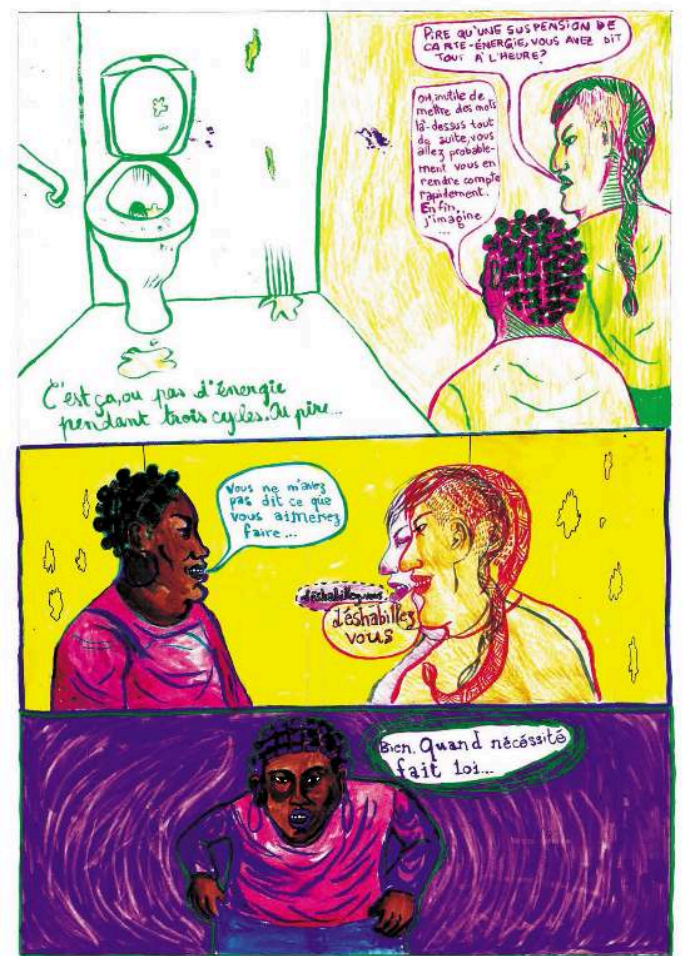


J'offre mon visage
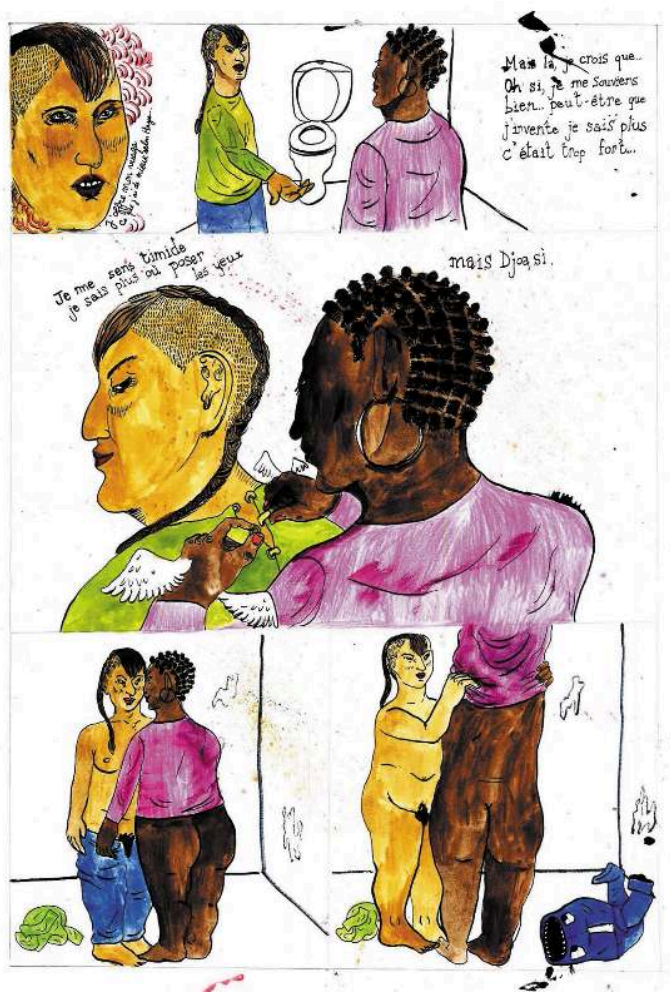

Votre poitrine... Magnifique...

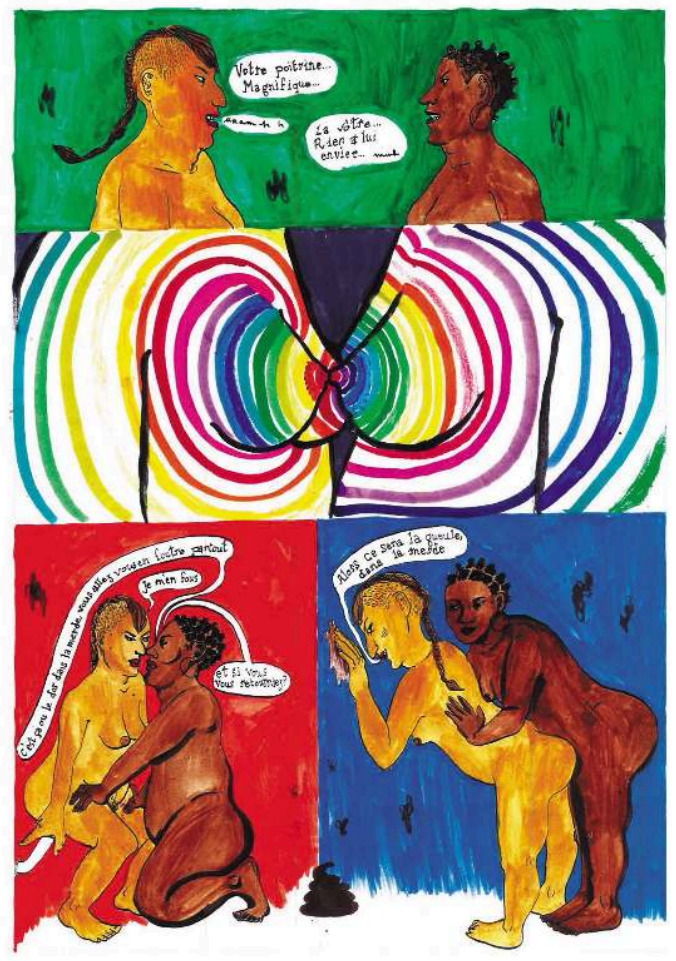


Les genoux qui CRAC !
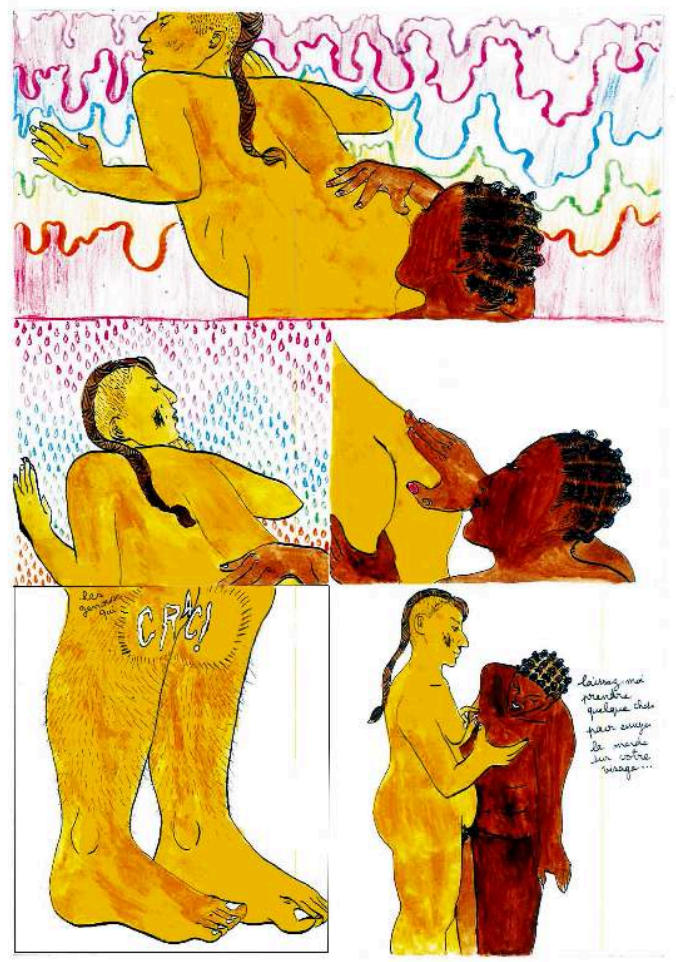

Je voudrais en finir
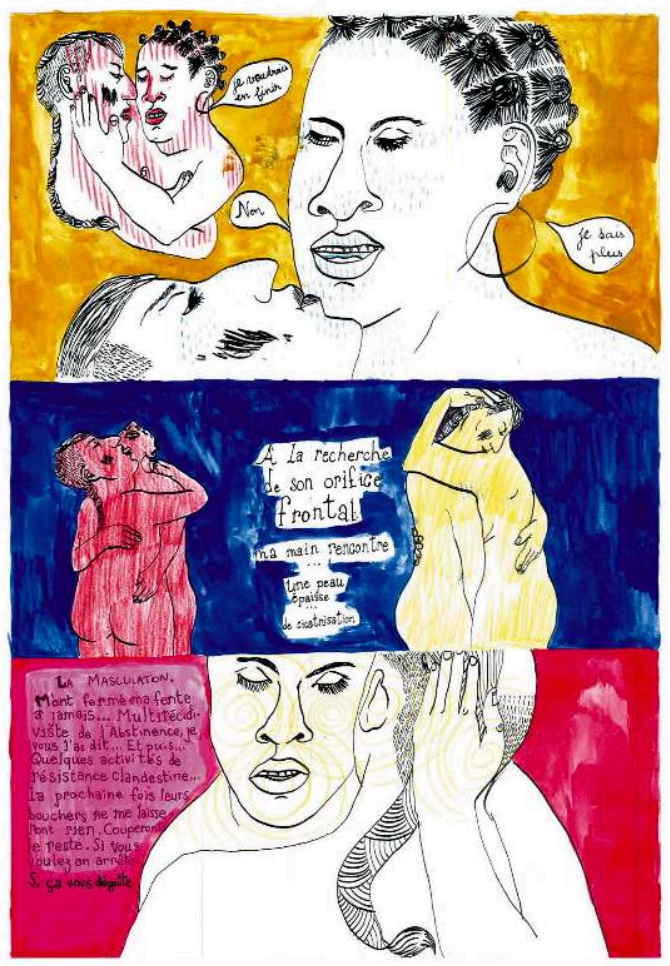


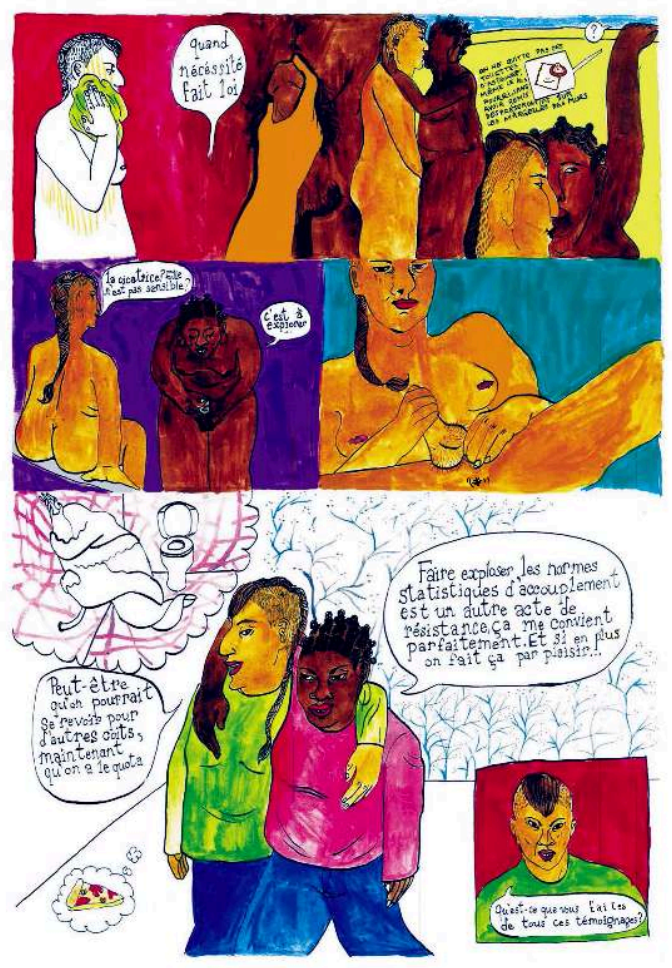

\section{Les Quotas, une nouvelle}

Ça s'annonçait mal, très mal.

10 Je n'avais pas mis les pieds dans ce genre d'endroit depuis ma mise en coupe avec Haye. Les codes de comportement qui devaient être en vigueur sur l'astronef allaient m'être devenus quasi inconnus. C'est terrible tout ce qu'une rupture vous enlève. J'avais l'impression de revenir dans une cité quittée cent cycles plus tôt. Une cité qui aurait beaucoup changé. Évidemment, durant tout ce temps! Inutile de rêver, les choses se transforment, elles n'attendent pas après vous.

Quant à ma cité intérieure... Des ruines et des cendres. La désolation. Appels sanglotés ne tirant de son sommeil que l'écho. Raser les murs, qui tombent en poussière.

Bref, pardon, aucun indice pour deviner quels étaient les codes actuels, voilà, j'étais sans repères, le dépaysement complet.

Il fallait en passer par là.

Si au moins Haye avait accepté qu'on fasse semblant encore quelques cycles. Bah, même ça, ça n'aurait servi à rien, puisque l'Inter avait commencé à contrôler les coupes établis comme tout le reste. Et elle envoyait ses tueurs jusque dans les coins de la Méga où autrefois elle fermait les yeux, où il y avait une certaine tolérance, on savait pouvoir contracter de l'union arrangée, de l'intercourse sur contrat - en plus d'éventuels désordres viraux, soit dit en passant. Même être en coupe, ça ne constituait plus une protection pour personne, plus une garantie, aucune assurance, même en coupe il fallait être actif et régulier et durer un temps bien déterminé, calculé selon le nombre de cycles de vie, sa position sur l'échelle sociale (une multitude de barreaux serrés les uns contre les autres tout en bas, puis un gouffre avant les derniers perchoirs). Selon 
l'origine, le revenu, etc. : je ne vous apprends rien, l'Inter rentre tous ces critères dans les Calculateurs pour déterminer le nombre d'actes de nature sexuelle qui vous sont prescrits. Et les derniers Calculateurs, à capteurs thermo-sensoriels, envoient aussitôt un message à l'Inter si vous les oubliez chez vous. Vous ne le saviez pas?

Est-ce qu'ici, entre vous, vous maintenez le même système de quotas de rapports, si je puis me permettre de poser la question?

Très bien, je poserai mes questions plus tard.

En tout cas, ça prouvait au moins une chose : que les contrôleurs de l'Inter, sous leurs apparences de butors, n'étaient pas des imbéciles finis qui se seraient laissé berner par la première furtive étreinte après un verre au comptoir, dans le brouhaha et les égosillements de leur musique de fondus.

Et tout le monde qui les prend pour des abrutis, moi comme les autres... Nous ne sommes toujours pas suffisamment évolués pour cesser de juger selon les apparences.

Bon, vu que je ne connaissais plus les codes, je savais que n'importe qui pouvait m'aborder, le tout-venant comme le raffiné, et la rencontre aléatoire, ça n'a jamais été ma tasse de thé, Haye me le reprochait suffisamment! Et tu ne sortirais pas de ta routine, de temps en temps !? Oublions tout ça... Cet épisode est... derrière moi...

En tout cas, j'avais vu juste : à peine reposé mon verre sur le comptoir métallique et claqué la langue pour sortir un dernier nano-blast de la substance de contrebande, qu'on s'avançait déjà ! Évidemment : on ne choisit pas un astronef de ce genre, sur un des anneaux les plus éloignés, quand on a froid aux yeux et les orifices serrés, « comme on va à confesse ", je crois qu'auraient dit les Anciens, tiens, c'est l'expression qui m'est revenue à ce moment-là mais je ne sais plus trop ce que ça veut dire, les Centres d'Instruction et les plaquettes de connaissance, c'est loin, tout de même. Ça fait un bail, je crois même que les Anciens auraient dit. J'aimais ça, étudier. Mais je n'ai pas pu me payer de quoi continuer.

18 Au moins, ça sonnait bien, "comme on va à confesse ", et j'avais besoin d'un peu d'assurance. Cela me tiendrait lieu de formule de protection. Presque une incantation.

Allez, ce n'était pas le moment de flancher, pour sûr je n'avais pas mis les pieds dans un tel gourbi depuis... je n'allais pas commencer à compter les cycles qu'avait duré mon coupe avec Haye! Je n'en menais pas large, mais c'était trop tard pour se dégonfler, l'autre n'avait pas l'air d'avoir l'intention de faire marche arrière :

- Bonsoir, je fonctionne sous le nom de Djoa. Vous êtes là pour la même chose que moi, je suppose?

Ouf, pour une approche directe, c'était une approche directe ! À moins que pendant ma période avec Haye, les entrées en matière aient beaucoup changé. Ce n'est pas cent cycles qu'on aurait cru, maintenant, mais dix mille...

Et voilà l'autre qui reprenait :

- J'ai déjà fait plusieurs tentatives ailleurs mais les sbires de l'Inter font régulièrement des rafles de contrôle, et désormais ce ne sont même pas leurs officiels: des mercenaires. Ce sont les pires ceux-là, ils ne ferment les yeux sur rien, ils sont animés d'un zèle de convertis! Et grassement payés, aussi. Bref, ils embarquent tout le monde, putes, clients, tout le monde, et je n'ai pas eu le temps de. La dernière fois, ils m'ont retiré ma carte d'approvisionnement-énergie pendant trois cycles. Et la fois précédente... Bref, ce n'est pas le pire qu'ils vous réservent, ils ne manquent jamais d'imagination, vous pouvez me croire... 
21 Le ton était las, j'ai trouvé. Ni colère ni révolte, non, rien que de la lassitude. Pas d'énergie pendant trois cycles, et il y avait eu pire... Multirécidiviste, donc. Ça me rappelait ma première Brimade, niveau 1 : logement réquisitionné pendant un tiers de cycle, retour à la case départ, au Communautaire, seul le Centre de Réhabilitation m'avait été épargné. Oh, personne ne s'était payé ma tête, on rame tous dans la même galère, mêmes injonctions pour tout le monde, à part évidemment pour les protégés de l'Inter.

Djoa, donc, multirécidiviste et avait connu pire... Voilà qui annonçait la couleur, ne faisait pas dans la dentelle et... Vous avez vu, j'ai de beaux restes de mes leçons sur les Anciens!

Bref, c'était à peine croyable, mais j'avais comme le désir qui s'éveillait. Quelque part. Encore timide, fragile sans aucun doute, mais quand même...

Haye n'avait pas tout emporté, alors, cela pouvait encore surgir, pousser dans mon désert dévasté, j'ai pensé.

- Bon, je continue la parlotte, puisque c'est moi qui ai commencé. On s'y prend comme vous voulez, je peux tout faire, j'aime tout. Ou plutôt...

Pause. Les moteurs arrière de l'astronef s'étaient déclenchés, complétant discrètement le brouhaha des voix des autres consommateurs. Quelques putes allaient et venaient, enfin, si c'étaient bien des putes, vu comme les choses avaient changé, je n'en aurais pas mis ma main au feu, c'était comme si je sortais tout juste du Communautaire et n'avais jamais rien vu...

25 J'ai parcouru du regard les bouteilles derrière le comptoir, poliment, comme ça se faisait avant, au cas où certains usages avaient encore cours. Pas mal d'étiquettes vieillies annonçaient le liquide de contrebande, les bouteilles vidées puis re-remplies... C'était vraiment un... lieu de perdition, encore une expression des Anciens qui me revenait, j'étais décidément en forme, c'était bon signe, je me suis dit.

Oh non, ce n'est pas dur de retrouver tous ces détails, j'ai la mémoire excellente.

Le plateau du comptoir avait perdu son lustre, les ronds laissés par les verres lui faisaient comme des ocelles, une maladie de peau, le méchant virus qui tue tant de monde. Cette pute qui faisait les cent pas derrière la plus grande table... Et si c'était un Contrôleur? J'en ai eu un frisson dans le dos, ce serait prudent de baisser le ton mais l'autre n'avait pas l'air méfiant, continuait son baratin, prenait sûrement mon silence pour un encouragement:

- Disons plutôt... j'en ai assez pris plein la figure pour ne plus avoir peur de... bref, rien ne me rebute, c'est vrai, je peux tout faire. Et...

Nouveau silence. C'était peut-être mon tour de dire quelque chose, mais quoi, on dit quoi quand on ne connait plus les codes !?

- Et... Bref, tout m'est égal.

D'un ton las, paupières à peine baissées. Eh bien, au moins, c'était sans ambiguïté ! Et mon désir à moi qui s'éveillait toujours, et voilà même qu'il s'ébrouait! C'était un des ces jours, trop rares, disait tout le temps Haye, à se demander si quelque chose chez moi trouvait grâce à ses yeux, c'était un de ces jours où j'allais me surprendre ? Voilà qui était cliché de chez cliché, même pour l'astronef le plus glauque... Et il fallait que je trouve à dire quelque chose, vite, avant que le désir ne se tire !

- Je m'appelle Kiméé. Allons aux chiottes.

Voilà, direct aussi, je me trouvais plutôt pas mal, sur ce coup, j'assurais, tiens !

- Déjà ? Vous ne voulez pas boire un verre? 
Ton sincèrement surpris. Eh, eh, tu ne t'attendais pas à ça, hein ? On relève les yeux, et moi j'ai offert tout mon visage, c'est ce que j'ai de mieux, disait Haye. La suite, d'un ton amusé :

- Si vous n'avez pas envie, on ne va pas y arriver.

Pause. Puis, encore :

- Mais ce sera sans importance.

Oh que si j'avais envie, j'avais même foutrement envie, et je l'ai dit comme ça, tant pis si ça fait vieux jeu, il y en a qui aiment :

- Si, j'ai envie, j'ai même foutrement envie de vous.

Car le désir avait fini de s'ébrouer, il était debout, tête haute, droite, regard franc, fier de sa puissance. Je l'ai vraiment pensé : voilà un jour où je vais me surprendre !

Djoa, avec un sourire: - Foutrement... Vous avez grandi chez des descendants d'Anciens, vous, ou alors vous avez bien potassé vos Plaquettes... Allons-y, précédezmoi.

J'ai caressé d'un doigt le comptoir, allez, debout, la trouille m'aurait serré le ventre si toute la place n'avait pas été prise par l'envie qui commençait à brûler, et tiens, non, la pute n'était pas un Contrôleur qui aurait été là pour nous, ou alors les codes avaient changé du tout au tout, mais ça aussi, c'était sans importance, je m'en foutais, ça ne comptait plus...

27 J'ai choisi les toilettes handicapées : je me sentais soudain en veine d'ironie, et tant pis pour celle, démesurée, toute-puissante, abusive de l'Inter, celle qui contraint tous les établissements, même sur un astronef aussi reculé que celui-là, à aménager des toilettes handicapées pour recevoir la clique d'éclopés, abîmés, massacrés, qui ressortent de ses salles d'interrogatoire. Le service après-vente des spécialistes de torture de l'Inter... Mais je m'en foutais, de leur humour noir de bourreaux, j'avais un dragon dans le ventre... le diable au corps, encore une expression... oh oui, j'étais en forme !!

Quand j'y repense...

L'endroit est vraiment dégueulasse, pas de raison, jusque sur les murs il y en a, et pourtant Djoa a l'air à l'aise. Il faut dire que c'est ça ou pas d'énergie pendant trois cycles, ou pire... Pire, justement, ça m'intrigue quand même, alors je demande :

- Pire qu'une suspension de carte-énergie, vous avez dit, tout à l'heure...

- Oh, inutile de mettre des mots là-dessus tout de suite, vous allez probablement vous en rendre compte rapidement. Enfin, j'imagine...

Et, nous ramenant à nos moutons :

- Vous ne m'avez pas dit ce que vous aimeriez faire.

Et moi je réponds, tranquille :

- Déshabillez-vous.

Le ton comminatoire, ma voix n'a pas l'habitude. Et pourtant, sans sonner faux, ça sonne... neuf. Oui, c'est ça, neuf. Mais il faut être honnête, je n'entends plus ma voix, à peine, à demi étouffées par les parois métalliques percées d'écrous et couvertes de zébrures, les voix des autres consommateurs restés englués devant leur verre, les pauvres. C'est que ça fait du bruit, du désir comme ça, ou plutôt, ça vide la tête. Non, ça rend sourd, voilà. Oui, c'est ça, ça rend sourd. Et j'ai répété, avec plus d'autorité encore :

- Déshabillez-vous.

- Bien. Quand nécessité fait loi... a dit Djoa en commençant, et en me regardant à la 
dérobée.

Mais là, je crois que... oh, si je me souviens bien... peut-être que j'invente, je sais plus, c'était trop fort... mais quand même je raconte, il faut bien... alors là j'ai offert tout mon visage, ce que j'ai de mieux, et ma main droite se tend vers Djoa qui s'avance, jambes nues déjà, enfin je crois, je me sens timide soudain, je sais plus où poser les yeux, mais Djoa, si, et les agrafes alambiquées de ma veste restent à défaire, apparemment la main droite de Djoa se sent des ailes... la gauche aussi, d'ailleurs... elle papillonne sur mes agrafes qui sautent l'une après l'autre, consentantes, et sur un coup de tête, voilà que sa gauche m'enlève mon pantalon, et le pantalon maintenant il nous observe depuis le sol sale, curieux, ça fait un moment qu'il n'a pas vu ça, il faut dire qu'avec Haye je ne connaissais plus que la disette... et j'ai l'impression d'avoir le regard de Djoa qui me remonte lentement les jambes, puis fait un bond jusqu'à ma poitrine...

- Votre poitrine... magnifique..., d'une voix qui glisse en même temps que nos chemises.

- La vôtre... rien à lui envier : l'autre voix, la mienne je crois, dans un sourire, et gémissements, déjà...

Djoa est tout près de moi, nos poitrines se rencontrent, souples, c'est l'astronef qui a bougé ? je recule contre la barre latérale d'appui destinée à ceux des interrogés par l'Inter qui ont pu conserver au moins un bras, Djoa m'attrape aux reins, c'est ça ou le dos dans la merde, vous allez vous en foutre partout, et ça me fait sourire, ce commentaire sobre en descendant lentement sur ses genoux, je m'en fous, je crois que j'ai dit, et si vous vous retourniez? Djoa demande, et moi je murmure, alors ce sera la gueule, dans la merde, une main sur le mur pour l'équilibre, il faudra que je l'essuie, mais sur quoi ? je m'en fous... l'autre main dans mon dos, dans les cheveux de Djoa, sa langue dans mon orifice, moi j'encourage, tant que j'y arrive encore, Djoa, la bouche pleine, on ne parle pas la bouche pleine, enseignaient les Anc...

Djoa se recule pour reprendre souffle, lentement je me retourne, je fais remonter Djoa à ma hauteur, ses genoux craquent légèrement... Djoa se redresse... seins contre seins...

Laissez-moi essuyer la merde sur votre visage, et Djoa se baisse pour ramasser sa chemise... mais moi je retiens ses seins, une main en serre, l'autre en pince mobile sur le téton durci et c'est à son tour de gémir...

Je voudrais en finir. Non. Je sais plus...

31 Ça veut dire quoi, finir? Je m'en fous, ma main-serre, voilà qu'elle descend, à la recherche de son orifice frontal, et elle rencontre... une peau épaisse...

De cicatrisation.

Les bourreaux.

Jamais ils ne manquent d'imagination, vous pouvez me croire.

Comme un hoquet, j'ai la compassion qui me saute à la gorge, pendant que Djoa s'excuse :

- Oui, ils m'ont masculé. Fermé ma fente à jamais... Multirécidiviste de l'abstinence, je vous l'ai dit... et puis... quelques activités de résistance clandestine... La prochaine fois leurs bouchers ne me laisseront rien. Couperont le reste. Si vous voulez, on arrête. Si ça vous dégoûte...

Si ça me dégoûte... De ma chemise j'essuie la merde sur mon visage. De ce jour, j'ai perdu le dégoût, et je m'en félicite...

Mon regard plongé dans le sien, sérieux. Mais ma main-serre, tandis que l'autre forme maintenant une coupe tendre sur son sein, ma main qui s'apprêtait à glisser sous son 
membre pour combler les chairs tendres et humides, impatientes, offertes, la petite bouche chaude mouillée que les mercenaires à la solde de l'Inter ont obturée, ma main remonte d'un pouce et saisit le membre qu'ils ont laissé. Jusqu'à la prochaine fois. Pour qu'il leur reste un endroit où punir à la prochaine sérieuse infraction.

Quand nécessité fait loi, je lui murmure à l'oreille, et Djoa, bras tendu, cherche en haut du mur de la cabine le préservatif qui devait y être, qui y était, car on ne quitte pas des toilettes d'astronef, même le plus pourri, sans avoir remis des préservatifs sur les margelles des murs - solidarité des marginaux, pour vous servir... Et tandis que la gaine légère s'enfile, je m'appuie des fesses contre la barre et je trouve à demander, faussement tranquille :

- La cicatrice ? Elle n'est pas sensible?

Et Djoa qui répond:

- C'est à explorer.

Après... peut-être que j'invente... comment se souvenir de ces moments-là ? après... un bras de chaque coté de ma tête... j'ai les jambes écartées... souffle qui s'élève... muscle du bras droit bandé pour pas se coller au mur merdeux... je plaque de la main gauche mon membre contre mon ventre pour laisser royal passage à Djoa qui plonge dans mes yeux un regard sourd... et le reste... et j'accompagne la cadence...

Peut-être qu'on pourrait se revoir pour d'autres coïts, maintenant qu'on a le quota... - j'ai murmuré. Faire exploser les normes statistiques d'accouplement est un autre acte de résistance. Ça me convient parfaitement - réponse de Djoa, d'une voix alanguie. Et si en plus, on fait ça par plaisir...

Je crois que c'est ce qu'on s'est dit. Je ne sais plus. J'avais la tête sur le côté comme une fleur fanée.

Qu'est-ce que vous en faites, de tous ces témoignages?

Ce texte a paru pour la première fois sur le carnet de recherche Reflecrire en juillet 2015 : https:// f.hypotheses.org/wp-content/blogs.dir/2591/

files/2016/01/

MLSchultze_Quotas_version_juil15.pdf

\section{Les Quotas, un texte de sertissage en trilogue}

\section{Moi c'est Ma.La.}

Les Quotas : nous voilà embarquées à trois dans ce délire, d'abord le texte, puis la BD de Jia, puis Jie qui retrouve le désir de publier la BD, puis tiens pourquoi pas le texte, puis tiens moi j'aimerais bien qu'on entende le texte mais c'est compliqué, des voix non genrées, et nous voilà embarquées à cinq dans le délire, deux lectrices ayant plongé !

Jie nous demande il y a quelques mois un petit texte d'accompagnement, qu'elle appelle, ça me séduit, un « texte de sertissage ».

Pour l'occasion je me remémore notre unique rencontre de travail, à Jia et à moi, sur sa $\mathrm{BD}$. Je me remémore le décalage que je constate, en découvrant sa BD, entre ce que je « vois » quand je relis le texte que j'ai écrit, et sa mise en images.

Je m'amuse de moi-même: ce n'est pas que dans le texte les personnages soient hermaphrodites qui me dérange; ce n'est pas que l'un de ces personnages ait été mutilé par la dictature en place - une masculation, qui consiste à fermer la fente. Non, ce qui me gêne, en réalité me dégoûte, c'est que les personnages se mettent de la merde 
partout!

Encore aujourd'hui, en regardant pour la nième fois les images de la BD de Jia, ce sont les traces de merde et d'urine sur les murs des toilettes qui me gênent, alors qu'en découvrant la $\mathrm{BD}$, j'avais été saisie par sa «crudité » dans la représentation des personnages, et les couleurs vives.

Alors que je l'y ai mise moi-même, la merde !

Franchement...

(Bon, sur la dernière image de Jia il y a aussi de la pizza, et ça, je n'en suis pas responsable...

Ça me rappelle ce que Jia avait écrit pour son premier texte de sertissage :

\begin{abstract}
Jia : «Pour moi, le fait de pas avoir de pronoms qui les assignent à un genre dans la nouvelle m'a donné envie de questionner nos représentations sur les corps, les attitudes, lues comme féminines ou masculines. Maintenant, j'ai même pas envie de me demander qu'est-ce que sont Kimee et Djoa, pour moi, ielles peuvent représenter des transgenres, des travesties, des intersexes, des gouines, des pédés, des bis... Ou complètement autre chose, vu que c'est un univers tellement éloigné du nôtre ».
\end{abstract}

Eh bien moi, 1/ je suis toujours dégoûtée par l'idée de baiser dans la merde 2/ je m'interroge sur la hiérarchie des mutilations prévues par la dictature de l'histoire 3/ avoir envie de manger de la pizza après le sexe...

$\mathrm{Au}$ départ, je voyais la masculation comme la transformation d'un hermaphrodite l'être «normal » de cet univers - en un « mâle », vu comme un individu porteur d'une verge produisant des spermatozoïdes. Pour la SFFF, l'univers des Quotas est ordinaire : ses hermaphrodites se reproduisent à des périodes données en passant soit en «dominance » femelle, soit en "dominance » mâle. Hors reproduction, tout le monde fait selon ses possibilités et ses désirs.

La masculation est un châtiment parce qu'elle vous prive de votre potentiel femelle. Ce qui m'intrigue, c'est que j'ai conservé l'ablation de la verge comme châtiment ultime pour ce personnage. Est-ce que l'Intergalactique en a fait une loi générale - au delà de l'émasculation, la mise à mort. On pourrait alors lire en filigrane : il est pire de perdre sa verge que sa fente. Ou bien les châtiments sont-ils "sur mesure ", l'Intergalactique évaluant au cas par cas ce qui peut être le pire pour sa victime (terme qui n'est pas le meilleur mais me permet de contourner le genre des noms).

S'il s'agit d'une loi générale, il y a dans mon texte une forme de machisme structurel: d'hermaphrodite, se retrouver femelle, bon, passe encore... mais ne plus pouvoir être mâle, quelle horreur !

Ce qui me fait penser à un autre commentaire de Jia :

\footnotetext{
Jia: «Avec du recul, je me demande si c'est symptomatique de quelque chose, d'avoir choisi de représenter des personnages noires dans cette histoire érotique, alors que les femmes noires sont déjà hypersexualisées dans les médias. J'arrive pas à savoir si c'est le résultat du racisme structurel qui travaille dans mes représentations ou si c'était juste une coïncidence de vouloir dessiner du porno et en même temps, de chercher des corps peu représentés en $\mathrm{BD} .$. En tout cas, il faudrait prolonger notre questionnement sur la raison de l'hypersexualisation des corps noirs, trans, la fétichisation de ces derniers ainsi que des femmes grosses dans notre société et questionner notre obsession pour les génitaux dans le sexe en général... Qu'est-ce qu'un sexe masculin? Un pénis de femme, ça existe pourtant!»
} 
41 Yo!

Je suis Jia, la partie dessinée de Quotas. Jia. Et je ne vois qu'une Noire.

Personnellement, le ressenti que j'avais du texte était que mes deux personnages étaient inspirés d'hommes. Et je ne suis pas certaine de voir deux femmes dans la BD de

2 Ça a commencé avec l'idée de faire des illustrations d'histoires pour enfants. Et puis Ma.La m'a passé son blog où elle met ses textes en ligne. Je suis tombée sur cette nouvelle qui m'a plu et qui était suffisamment courte pour que je sois sûre de la terminer. C'est mon premier projet de BD mené à terme. Avant ça, j'ai fait un zine avec des dessins d'angoisses, mais y avait pas de scénario.

Pour traduire Quotas en BD, Ma.La m'a laissé jouer sans limites. Au début c'était un peu déconcertant parce qu'il n'y a pas de description physique des personnages ni des lieux. Et il y a pas mal d'expressions inventées, qui laissent beaucoup de place à l'interprétation. Par exemple "nanoblast » ou bien « centre de réhabilitation ». J'y ai mis un sens qui m'a paru refléter le côté post-apocalyptique de l'univers de l'Inter. Il y a une histoire marrante à propos du «Communautaire », j'suis tombée sur une photo de prison pour femmes des années 30 au moment de dessiner cette case (page 5). Cette photo apparaît en couverture du livre Les Écoles de préservation pour les jeunes filles publié chez L'Arachnéen. (merci ma cousine, de m'avoir providentiellement envoyé ce bouquin).

Ici Jie.

Je n'étais pas là quand Jia et Ma.La se sont rencontrées et qu'est née l'idée de cette déclinaison d'histoire de texte à bédé. Un jour Jia m'a montré la bédé, en me disant que c'était "tiré » d'un texte de Ma.La. Ma.La qui avait été ma prof à la fac, et qui intervenait dans le cursus que suivait Jia. Jia que je connaissais des milieux anarchopunk et avec qui nous parlions beaucoup de genre et de gens. Et toutes deux, chemin faisant, qui étaient devenues des copines. GLAD! était en train de se monter et se cherchait déjà sur la frontière entre parole académique et parole exploratoire. Cette bédé, elle était juste sur cette frontière, une narration qui éclatait le genre tout en parlant sexe, et trois personnes dans les marges de l'université qui ne se résolvaient pas encore tout à fait soit à la quitter, soit à y entrer de plain-pied. C'était plié : il fallait publier la bédé de ces deux-là dans GLAD!

Puis, bien plus tard, quand Ma.La m'a parlé de la bédé, elle m'a dit que cette bédé, elle ne lui appartenait pas tellement, que c'est Jia qui s'en était saisie. Et que c'est GLAD! qui voulait la publier. Qu'avait-elle à voir avec tout ça ? Elle, après tout, elle n'avait fait qu'écrire un texte... Alors, c'était quoi cet objet ? C'était qui les auteur.es ? Puis, on a continué à parler, et on s'est dit, enfin non, elle m'a dit que son texte, elle l'imaginait dans l'oralité. Et si on enregistrait? Mais il faudrait des voix pas trop genrées. Est-ce qu'on peut convenablement faire lire ce texte à des pré-ado ? Bon, là intervient Ma.Ca, la phonétique à votre service. On trouve Vlan et Épicène, qui veulent bien enregistrer. Voilà, maintenant, on a un texte, une bédé, un enregistrement audio et une revue, et on ne sait toujours pas trop qui fait quoi. Puis bon, les lecteurices, qu'est-ce qu'illes vont y comprendre? Trois déclinaisons d'une même histoire, peut-être qu'il faut expliquer? Tracer une sorte de généalogie? ok, mais on explique quoi ? Comment? On fait un texte à trois voix ? Voilà. On aurait peut-être voulu mêler ces trois voix davantage, mais c'est comme ça: on voulait faire un texte pour démêler et on s'emmêle, ou plutôt 
l'inverse, là où on voulait démêler, on a rajouté une couche de non-auteures (nonnes auteures ?), là où on voulait mêler nos voix, on superpose, on aligne. Bon alors quoi. Si on laissait comme ça?

Alors Jia a dit, pour finir, «J'avais aussi envie de vous dire combien des BDs comme celle-ci peuvent être considérées comme dangereuses au Brésil. Je vais vous donner des anecdotes de l'actualité : il y a un Musée célèbre à São Paulo où des vieilles toiles ont été recouvertes de tissus noirs parce qu'elles représentent des gens à poil! Il y a des peintres et performeurs qui ont été condamnés parce que leur travail contenait de la nudité et il y a une exposition qui a été interdite qui s'appelait "Queer museum", accusée de montrer de la zoophilie et de la pédophilie, alors qu'il n'y avait que des scènes homos !! Ah, et une dernière énormité : Judith Butler a été agressée quand elle est passée au Brésil, donner une conférence. Les manifestants hurlaient Brûlez la sorcière ! en même temps qu'ils foutaient le feu à une poupée à l'effigie de la philosophe.

Bref, parler de sexe c'est mal vu ici et ça donne encore plus envie de traduire la BD, vu que ça a été dessiné au Brésil.

Je voulais aussi faire part d'un néologisme en portugais que je trouve rigolo : il y a un mot inventé pour dire anus qui ressemble à un mélange entre le mot pour dire foune et le mot cul... comme on pourrait traduire par coune, c'est comme ça que certaines meufs trans ou travesties aiment parler de leur fente..."

47 Alors finalement, ce texte de sertissage, il est peut-être bien comme ça. Parce qu'il permet d'inventer coune. Pour le reste, on vous laisse lire, regarder, écouter.

\section{NOTES}

1. Le texte a paru pour la première fois sur le carnet de recherche Reflecrire en juillet 2015 : https:/f.hypotheses.org/wp-content/blogs.dir/2591/files/2016/01/

MLSchultze_Quotas_version_juil15.pdf

2. https://reflecrire.hypotheses.org/321

3. https://reflecrire.hypotheses.org/46

\section{RÉSUMÉS}

Ce qu'on voit se passe sur un astronef miteux de fond de galaxie, où l'Intergalactique, le pouvoir totalitaire en place, traque ceux qui ne se soumettent pas à ses lois. Parmi celles-ci, l'obligation de se livrer à des coïts dont le nombre par période de temps est fixé par l'Inter. Le choix de avec qui, comment et surtout à quel rythme vous avez des relations sexuelles, dans cet univers, ne 
vous appartient pas.

Ce récit est décliné sous la forme d'une bédé, d'un enregistrement audio et d'une nouvelle, trois manières (graphique, audio et textuelle) de tenter de déjouer le genre et la sexualité de Kimée et Djoa, les personnages, et de leur univers. Ces trois variations sur le thème de l'Inter sont accompagnées d'un court texte introductif et assortis d'un texte de sertissage qui revient sur la création de ces trois objets.

What you will see happens aboard a shabby spaceship, at the edge of a galaxy. The Intergalactic, the totalitarian power in place, stalks those who don't obey to its rules. Among these rules, the obligation to engage in coitus, with a number of coitus per period fixed by the Inter. In this universe, the choice of whom you have sexual relationships with, how and at which rate doesn't belong to you anymore.

This narrative is presented in three different forms: a comic, an audio recording and a novel, three ways (graphic, audio and textual) as three attempts to thwart Kimée and Djoa's gender and sexuality-the characters, and their universe. These three variations on the theme of the Inter are introduced by a short introduction, and followed by a "seaming text", which genealogically recounts the creation of these three

\section{INDEX}

Thèmes : Créations

Keywords : sci-fi, compulsory sexuality, androgynous characters, biopolitics, multimodal creation

Mots-clés : science-fiction, sexualité obligatoire, personnages androgynes, biopolitique, création multimodale

\section{AUTEURS}

\section{MARIE-LAURE SCHULTZE}

MLS ne sait ni coudre, ni chanter, mais fait vraiment bien les crêpes et la mousse au chocolat. Après 25 ans de services aussi loyaux que possible auprès de l'Université française, elle s'occupe désormais exclusivement de ses affaires d'écriture et d'animation d'ateliers. Une partie de son travail est visible ici : https://reflecrire.hypotheses.org/author/reflecrire

\section{SUJAREI TALI}

Colectivo Entulho https://entulhoviralatas.tumblr.com/

Ju, 23 ans, dessine en autodidacte, cuisine, coud, chante, fait du dessin animé... Dans le désordre et contre l'ordre établi. 